\title{
The Effect of Alogliptin and Metformin Combination Therapy in Type 2 Diabetes: A Pilot Study
}

\author{
Miyako Kishimoto $^{\mathrm{a}, \mathrm{b}, \mathrm{d}}$, Kaori Inoue ${ }^{\mathrm{a}}$, Mitsuhiko Noda ${ }^{\mathrm{b}, \mathrm{c}}$
}

\begin{abstract}
Background: As type 2 diabetes is characterized by both insulin resistance and impaired insulin secretion, oral anti-diabetic treatments including dipeptidyl peptidase-4 inhibitors, which stimulate insulin secretion, and biguanides, which enhance insulin sensitivity should be considered. Therefore, we performed a pilot study using a combination therapy with agents from both of these classes of drugs. We selected alogliptin and metformin as representative drugs and evaluated their combinational efficacy in patients with type 2 diabetes.
\end{abstract}

Methods: Continuous glucose monitoring (CGM) was performed throughout the study for two patients with type 2 diabetes and one control subject. First, the subjects received no medication (the 5-day washout period), and later, all subjects received three patterns of medication: alogliptin alone, alogliptin and metformin coadministration, and metformin alone. Blood was sampled before and $1 \mathrm{~h}$ after breakfast and lunch on representative days during each treatment condition.

Results: The CGM results indicated that combination of alogliptin and metformin attenuated the escalation and fluctuation of glucose levels. The patterns of insulin and glucagon secretion with alogliptin alone, alogliptin and metformin co-administration, and metformin alone varied among subjects. When alogliptin and metformin were

Manuscript accepted for publication October 25, 2013

${ }^{\mathrm{a}}$ Department of Diabetes, Endocrinology, and Metabolism, Center Hospital, National Center for Global Health and Medicine, Tokyo, Japan

${ }^{\mathrm{b}}$ Diabetes and Metabolism Information Center, Diabetes Research Center, Research Institute, National Center for Global Health and Medicine, Tokyo, Japan

${ }^{c}$ Department of Diabetes Research, Diabetes Research Center, Research Institute, National Center for Global Health and Medicine, Tokyo, Japan

${ }^{\mathrm{d}}$ Corresponding author: Miyako Kishimoto, National Center for Global Health and Medicine, 1-21-1 Toyama, Shinjuku-ku, Tokyo 162-8655, Japan.Email: mkishimo@hosp.ncgm.go.jp

doi: http://dx.doi.org/10.4021/jem196e co-administered, glucagon-like peptide-1 (GLP-1) levels $1 \mathrm{~h}$ after lunch were higher in all subjects compared to those at any other time point. Postprandial glucose-dependent insulinotropic peptide (GIP) levels varied according to medication and the subject.

Conclusions: Thus, CGM results revealed that a combination of alogliptin and metformin effectively reduced postprandial glucose fluctuation and stabilized blood glucose levels. The study subjects exhibited completely different response patterns of insulin, glucagon, GLP-1, and GIP with medications alone or in combination, suggesting that individual hormone-dependent glycemic responses to these drugs are complicated and multifactorial.

Keywords: Metformin; DPP-4 inhibitor; Glucagon-like peptide-1; Glucose-dependent insulinotropic peptide; Continuous glucose monitoring

\section{Introduction}

Incretins such as glucagon-like peptide-1 (GLP-1) and glucose-dependent insulinotropic polypeptide (GIP) are released from enteroendocrine cells and are known to enhance insulin secretion $[1,2]$, but are rapidly inactivated by the enzyme dipeptidyl peptidase-4 (DPP-4) [3]. DPP-4 inhibitors such as alogliptin are a new class of anti-diabetic agents that increase circulating GLP-1 and GIP levels by inhibiting DPP-4 enzymatic activity. GLP-1 and GIP improve hyperglycemia in a glucose-dependent manner by increasing serum insulin and decreasing serum glucagon levels in patients with diabetes [4]. Biguanides such as metformin are another class of anti-diabetic agents that curtail hepatic glucose output by inhibiting gluconeogenesis and also increase insulinstimulated glucose uptake in muscle and fat $[5,6]$. Insulin resistance and impaired insulin secretion are two major features of type 2 diabetes, and biguanides and DPP-4 inhibitors lower glucose levels using different but complementary mechanisms. Therefore, combination therapies using these agents are worth considering. In fact, metformin is increasingly being used in combination with various DPP-4 inhibitors [7-10].

In this pilot study, we evaluated the effects of alogliptin 
Table 1. Subject Characteristics

\begin{tabular}{llll}
\hline & Case 1 & Case 2 & Control \\
\hline Age (years) & 57 & 58 & 48 \\
Sex & Male & Male & Female \\
BMI $\left(\mathrm{kg} / \mathrm{m}^{2}\right)$ & 22.1 & 26.1 & 23.1 \\
Diabetes duration (years) & 5 & 5 & None \\
Treatment prior to study & Alogliptin & Metformin & None \\
HbA1c $(\%)$ & 7.3 & 7.4 & 5.7 \\
\hline
\end{tabular}

BMI, body mass index; HbA1c, hemoglobin A1c.

and metformin alone and in combination on changes in blood glucose levels by using a continuous glucose-monitoring system (CGMS) [11-13]. Moreover, the effects of these agents on changes in insulin, glucagon, active GLP-1, and total GIP levels in two patients with type 2 diabetes and a normal control subject were determined.

\section{Material and Methods}

\section{Subjects}

The baseline characteristics of the two Japanese in-patients with type 2 diabetes and one Japanese control subject included in this study are summarized in Table 1. All study participants signed informed consent forms and were free to withdraw from the study at any time. The study was conducted in accordance with the Declaration of Helsinki of 1964, as revised in 2008, and guidelines for good pharmacoepidemiology practice.

\section{Procedure}

\section{CGMS}

All anti-diabetic drugs for the two patients were washed out at least for 5 days before study initiation. The two patients were admitted to the diabetes ward at the National Center for Global Health and Medicine and equipped with a CGMS device (Medtronic MiniMed, CGMS-GOLD, USA). The patients were monitored for 1 day before the study until the end of the study period. The CGMS soft sensor was changed at the appropriate times according to the manufacturer's instructions. Glucose levels were measured with a self-monitoring blood glucose device (Nipro Stat Strip XP, Nipro, Japan) at least 4 times per day to calibrate the CGMS. The recorded data were analyzed with CGMS Solutions software.

\section{Calorie intake}

Calories of each breakfast and lunch throughout the study period were determined considering the age and body mass index (BMI) of the subject. The mean calories contained in the breakfasts of patients 1 and 2 and the control subject were 393, 390, and $456 \mathrm{kcal}$, respectively; and those in lunch were 593, 592, and $519 \mathrm{kcal}$, respectively.

\section{Sample collection and analysis}

On the first day of the study, the subjects had fasted overnight for $14 \mathrm{~h}$. An intravenous line was inserted into the forearm vein of each subject that was flushed with sterile $0.9 \% \mathrm{NaCl}$ solution for repeated blood sampling. Blood was drawn before and $1 \mathrm{~h}$ after breakfast and lunch (meals were ingested within 10 - $15 \mathrm{~min}$ ) to measure serum insulin, plasma glucagon, active GLP-1, and total GIP. Blood samples for the determination of active GLP-1 were collected into chilled BD P700 tubes containing spray-dried dipotassium EDTA as anticoagulant and proprietary DPP-4 protease inhibitor (Becton Dickinson, Franklin Lakes, NJ, USA). GLP-1 concentrations were measured using an enzyme-linked immunosorbent assay (ELISA) kit (Millipore Corp., Billerica, MA, USA). Plasma concentrations of amidated GLP-1 (7-36) and (7-37) were measured using an antibody highly specific for the $N$-terminus of GLP-1 that does not react with GLP-1 (936), GLP-2, or glucagon. The detection limit of the ELISA was $2 \mathrm{pmol} / \mathrm{L}$, with intra- and inter-assay coefficients of variation $(\mathrm{CVs})$ of $2.6-6.0 \%$ and $7.1-9.8 \%$, respectively. Total GIP was measured using a human GIP ELISA kit (Millipore Corp., Billerica, MA, USA) that has $100 \%$ cross reactivity with human GIP (1-42) and GIP (3-42) with a detection limit of $1.8 \mathrm{pmol} / \mathrm{L}$ and intra- and inter-assay $\mathrm{CVs}$ of $4.8-6.3 \%$ and $2.2-5.0 \%$, respectively. Serum insulin was measured using a chemiluminescent enzyme immunoassay. Blood samples for glucagon measurements were collected in tubes containing 

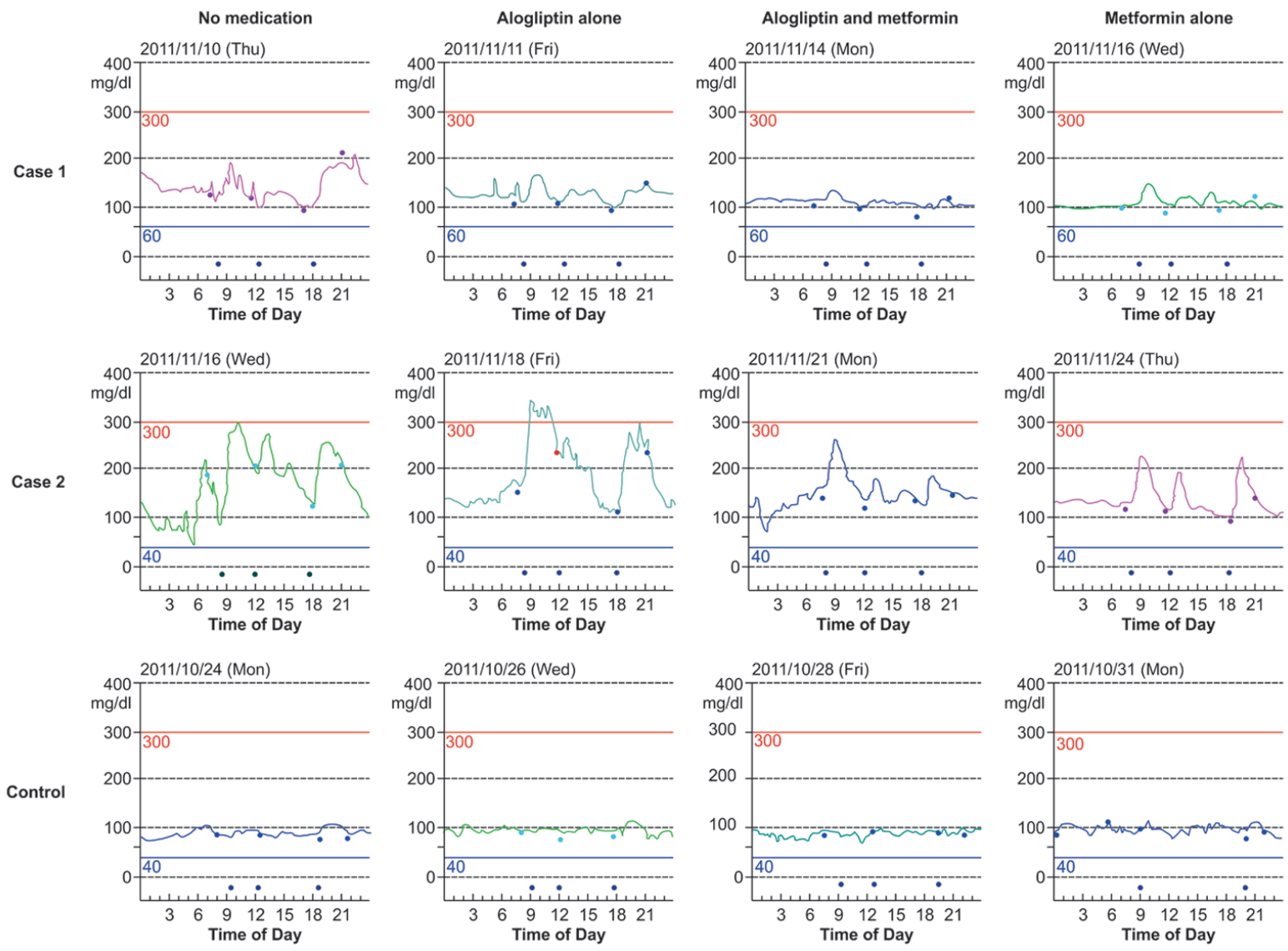

Figure 1. Continuous glucose monitoring (CGM) results. CGM results of patients with diabetes in cases 1 and 2 and that of the control subject obtained when no medication, alogliptin alone, alogliptin and metformin co-administration, and metformin alone were administered. The signs at the bottom of the figures indicate the time of the meals (In the figure for the control patient, the sign indicating lunch on the day when metformin alone was administered is missing). The signs on the lines of each figure indicate the times of calibration.

disodium EDTA plus aprotinin and analyzed by a double-antibody radioimmunoassay. All sample measurements, except those for active GLP-1, were performed by SRL Inc. (Tokyo, Japan). Active GLP-1 levels were measured by Mitsubishi Chemical Medience Corporation (Tokyo, Japan).

\section{Medications}

Prior to study initiation, the subjects received no medication for at least 5 days. CGM was initiated on day 0 , and the first blood samples were collected on day 1 (for baseline data). Subsequently, alogliptin $(25 \mathrm{mg})$ was administered before breakfast on days 2 and 3. On day 4, metformin $(250 \mathrm{mg}$ twice daily) was given shortly after breakfast and dinner, in addition to the alogliptin. Subjects were cautioned about gastrointestinal symptoms as a possible adverse effect of metformin. On day 5 , the metformin dosage was increased to $250 \mathrm{mg}$ thrice daily (shortly after each meal), in addition to the alogliptin. On day 6 or 7, alogliptin was withdrawn, and the subjects continued to receive metformin alone for 3 days. Blood was sampled on each day at the same time and using identical sampling methods.

\section{Results}

During the study period, the subjects experienced no gastrointestinal symptoms caused by the medications, and CGM was completed without any complication. The blood glucose levels measured by CGM are shown in Figure 1. In case 1, blood glucose fluctuations were attenuated after alogliptin administration compared to that during no medication; this attenuation was even more pronounced with alogliptin and metformin co-administration. The attenuation of blood glucose fluctuations persisted after alogliptin was withdrawn and metformin alone was administered. In case 2, the fluctuations of blood glucose levels were not different with alogliptin alone compared to that during no medication. However, during metformin and alogliptin co-administration, the fluctuation was attenuated and glycemic control improved. The attenuation of blood glucose fluctuations persisted even after alogliptin was withdrawn and metformin alone was giv- 
Table 2. Twenty-Four-Hour Glucose Levels Measured by Continuous Glucose Monitoring

\begin{tabular}{llcc}
\hline & Case 1 & Case 2 & Control \\
\hline Glucose $(\mathrm{mg} / \mathrm{dL})($ mean $\pm \mathrm{SD})$ & & & \\
No medication & $144 \pm 26$ & $170 \pm 66$ & $93 \pm 9$ \\
Alogliptin alone & $132 \pm 14$ & $190 \pm 64$ & $100 \pm 7$ \\
Alogliptin + metformin & $116 \pm 7$ & $151 \pm 32$ & $88 \pm 7$ \\
Metformin alone & $110 \pm 11$ & $136 \pm 29$ & $99 \pm 9$ \\
\hline
\end{tabular}

SD, standard deviation.

en. There were no remarkable fluctuations of blood glucose levels in the control subject. The accuracy of these measurements was reflected by the means and standard deviations (SDs) of CGM measurements (Table 2).

The time courses of insulin, glucagon, active GLP-1, and total GIP levels before and $1 \mathrm{~h}$ after breakfast and lunch are summarized in Figures 2-4. In case 1, alogliptin alone, alogliptin and metformin co-administration, and metformin alone attenuated the postprandial increase in plasma insulin levels compared to that during no medication. In case 2 , the postprandial increases in plasma insulin levels in all conditions were quite similar. In the control subject, the postprandial insulin levels $1 \mathrm{~h}$ after breakfast were higher on metformin alone, similar on alogliptin alone, and lower on alogliptin
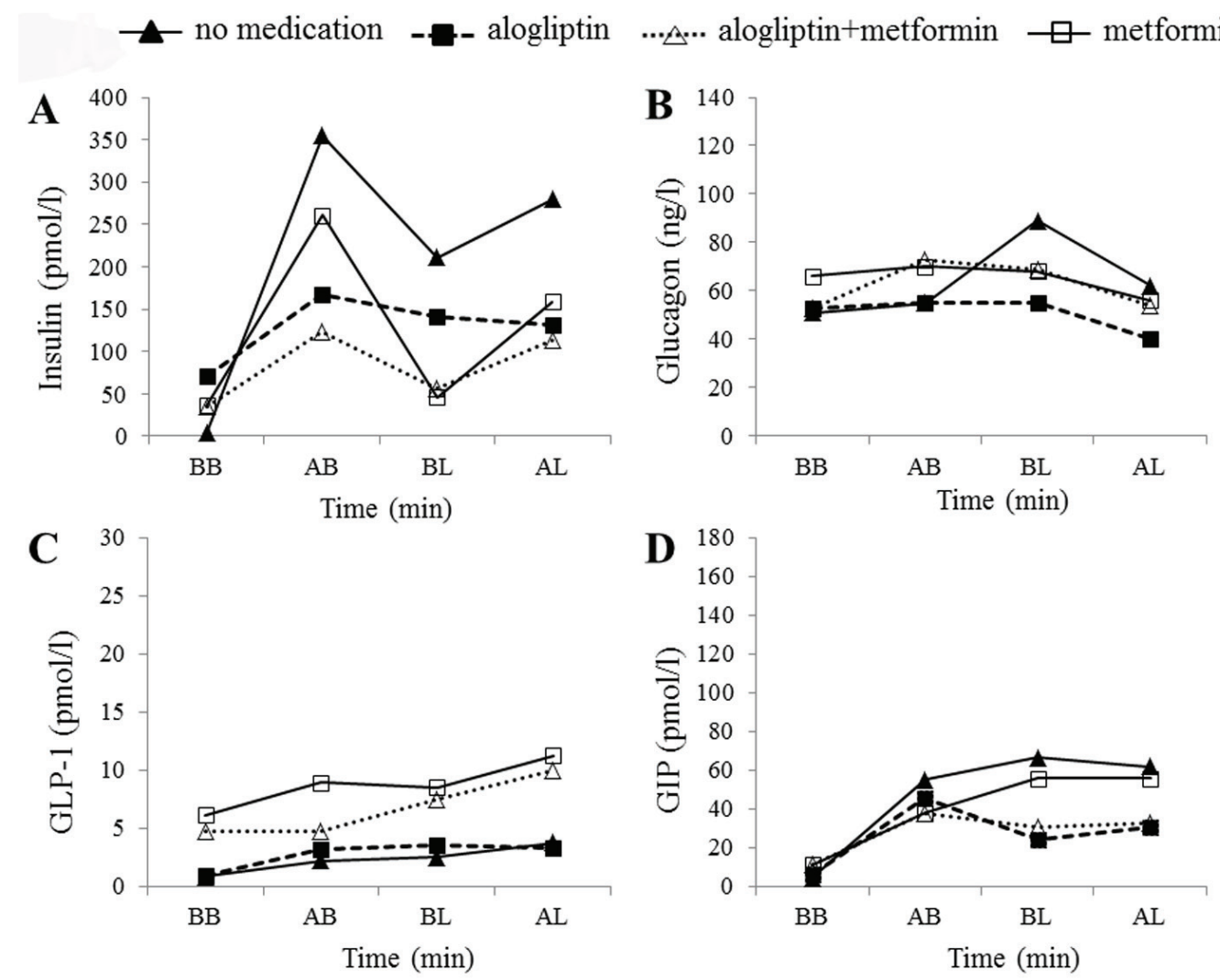

Figure 2. Hormonal changes in case 1. Time courses of plasma insulin (A), glucagon (B), active glucagon-like peptide-1 (GLP-1) (C), and total glucose-dependent insulinotropic peptide (GIP) (D) levels before breakfast (BB), $1 \mathrm{~h}$ after breakfast (AB), before lunch (BL), and $1 \mathrm{~h}$ after lunch $(\mathrm{AL})$ in the patient in case 1. Filled triangles, filled squares, open triangles, and open squares indicate no medication, alogliptin alone, alogliptin and metformin co-administration, and metformin alone, respectively. 

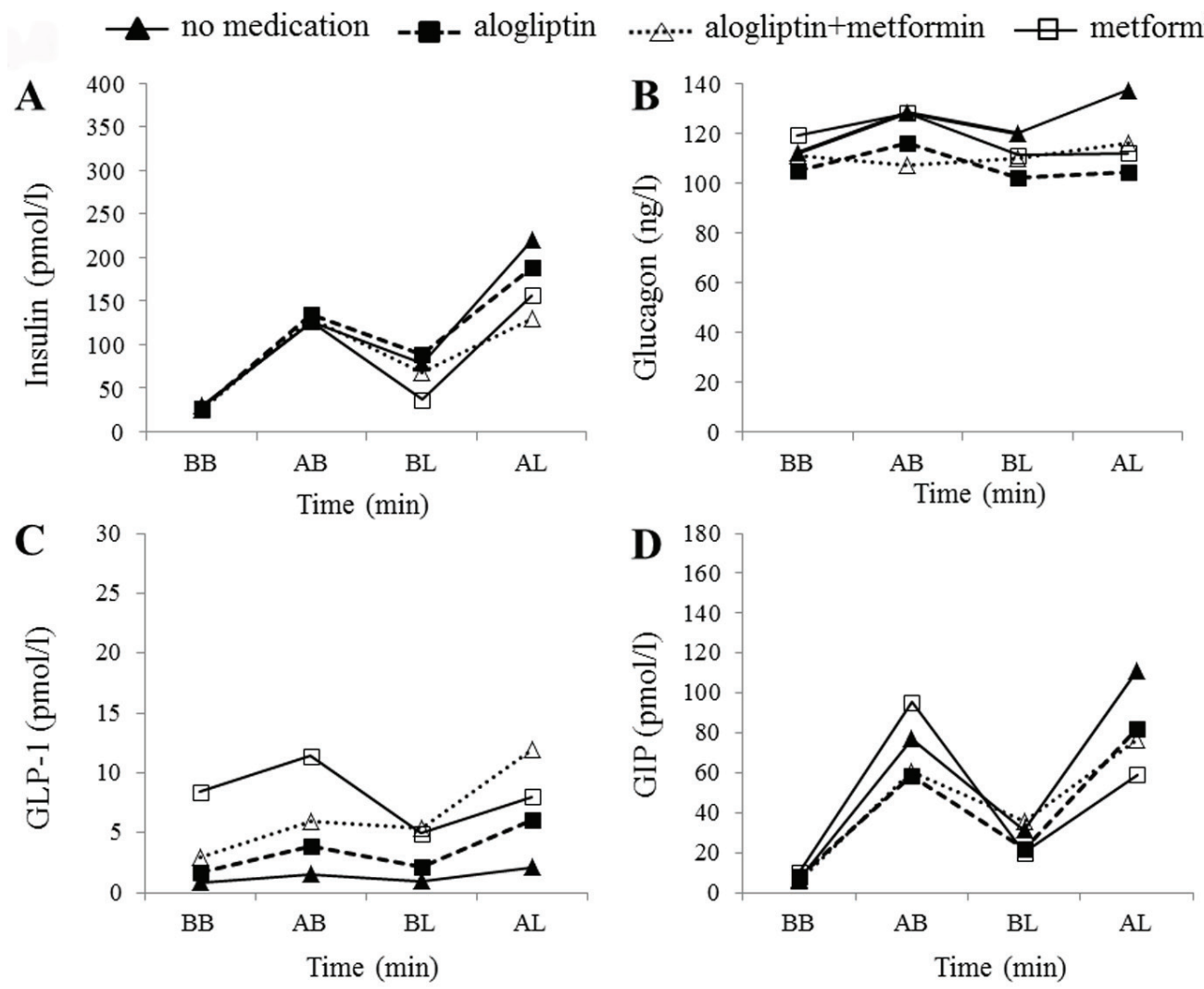

Figure 3. Hormonal changes in case 2. Time courses of plasma insulin (A), glucagon (B), active GLP-1 (C), and total GIP (D) levels before breakfast $(B B), 1 \mathrm{~h}$ after breakfast $(A B)$, before lunch $(B L)$, and $1 \mathrm{~h}$ after lunch $(A L)$ in the patient in case 2 . Filled triangles, filled squares, open triangles, and open squares indicate no medication, alogliptin alone, alogliptin and metformin co-administration, and metformin alone, respectively.

and metformin co-administration, as compared with that during no medication. In cases 1 and 2, glucagon levels before and $1 \mathrm{~h}$ after lunch were lower when alogliptin alone, alogliptin and metformin co-administration, and metformin alone were administered compared to that when no medication was given. In the control subject, glucagon levels at all time points were higher when alogliptin alone, alogliptin and metformin co-administration, and metformin alone were provided compared to that when no medication was given. In all subjects, postprandial active GLP-1 levels were higher when alogliptin and metformin co-administration and metformin alone were administered as compared to that when no medication was given. In the control subject, postprandial GLP-1 levels were remarkably higher when alogliptin alone, alogliptin and metformin co-administration, and metformin alone were administered compared to that when no medication was given; GLP-1 elevation, especially $1 \mathrm{~h}$ after lunch, was most prominent when alogliptin and metformin were coadministered. After alogliptin administration with or without metformin, the GIP levels after lunch reduced compared to that observed when no medication was given. When on metformin alone, the GIP levels after breakfast were higher in case 2 and the control subject, and those after lunch, were lower in all subjects.

\section{Discussion}

In this study, the administration of alogliptin alone reduced glycemic fluctuation in case 1 but not in case 2 of our patients with diabetes. However, this effect was more pronounced in both cases when alogliptin was co-administered with metformin. In cases 1 and 2, glycemic control was not worsened after alogliptin was withdrawn and metformin alone was administered continuously. The maintenance of strict dietary control during hospital stay may have contributed to this. Alternatively, the fact that insulin resistance rather than insulin deficiency was predominant in the two patients may explain why metformin worked better than alogliptin.

Metformin increases plasma active GLP-1 in humans [14-18]. There are two possible mechanisms for this effect: 1) metformin inhibits DPP-4 [19], or 2) metformin enhances GLP-1 secretion. The first mechanism is controversial because some studies refute any direct effect of metformin on DPP-4 $[15,20]$. The second mechanism is supported by the work of Maida et al who reported that metformin actually 

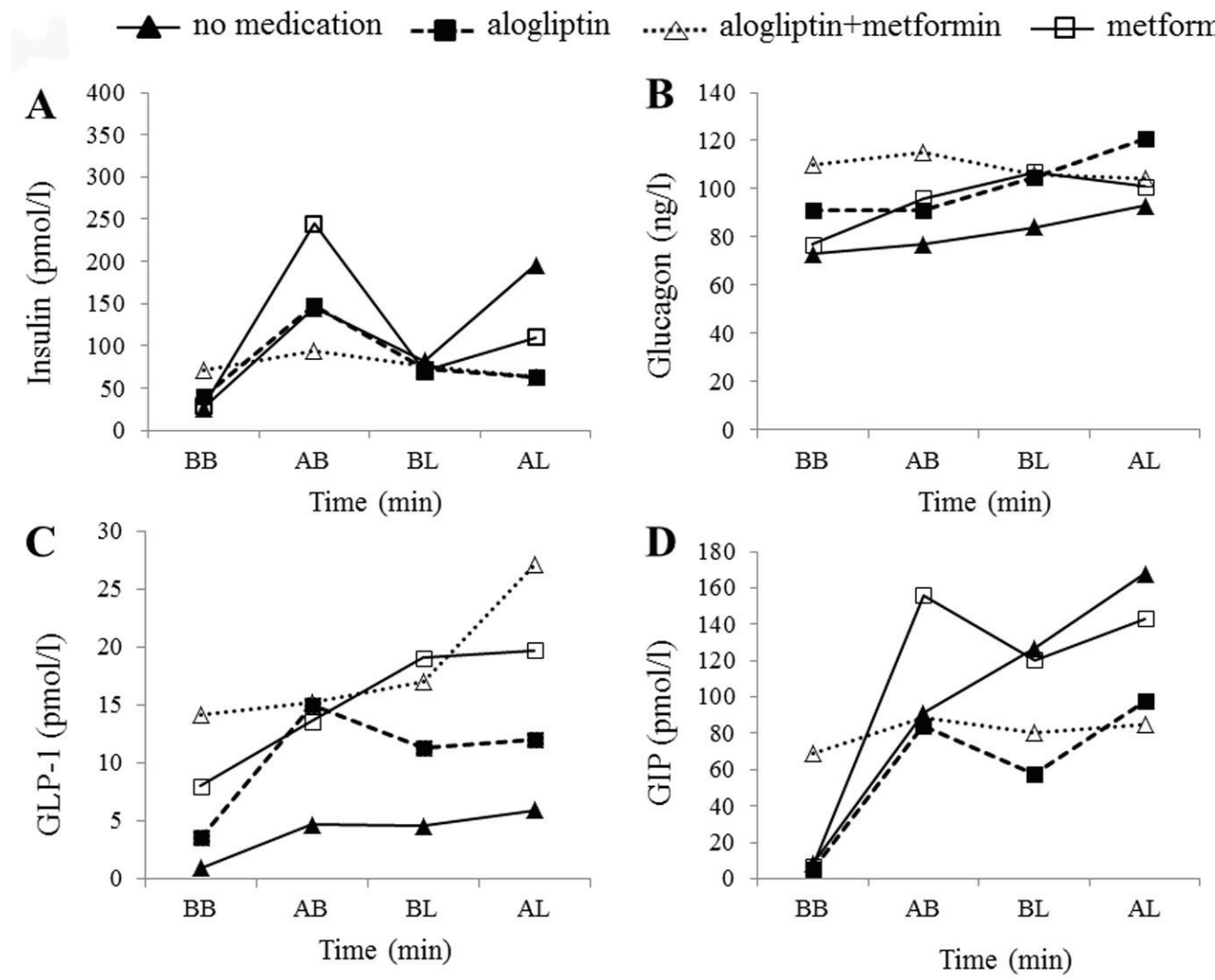

Figure 4. Hormonal changes in the control subject. Time courses of plasma insulin (A), glucagon (B), active GLP-1 (C), and total GIP (D) levels before breakfast (BB), $1 \mathrm{~h}$ after breakfast $(A B)$, before lunch (BL), and $1 \mathrm{~h}$ after lunch $(A L)$ in the control subject. Filled triangles, filled squares, open triangles, and open squares indicate no medication, alogliptin alone, alogliptin and metformin co-administration, and metformin alone, respectively.

increased plasma levels of GLP-1 in mice, enhanced the expressions of the genes encoding the receptors for both GLP-1 and GIP in mouse islets, and increased the effects of GIP and GLP-1 on insulin secretion from $\beta$ cells [21]. Interestingly, these incretin-sensitizing effects of metformin appear to be mediated by a peroxisome proliferator-activated receptor $\alpha$-dependent pathway involving adenosine monophosphateactivated protein kinase $[14,21]$. In addition, Migoya et al [22] reported that metformin did not inhibit DPP-4, but rather enhanced precursor preproglucagon expression in the large intestine, resulting in increased total GLP-1 concentrations.

To assess the drug-induced hormonal changes more precisely, we also measured insulin, glucagon, GLP-1, and GIP levels after drug administration. In case 1, all treatments attenuated the postprandial increment in insulin compared to that during no medication, which may be explained by an induced decrease in blood glucose levels. In case 2, the postprandial insulin levels $1 \mathrm{~h}$ after breakfast did not differ in response to any treatment compared to that during no medication; the reason for this is unclear. Because DPP-4 inhibitors are expected to increase GLP-1 levels, leading to restrained glucagon secretion, we anticipated suppressed glucagon levels, especially with alogliptin administration. This phenomenon was observed in the two patients with type 2 diabetes, but not in the control subject. Metformin administration increased postprandial active GLP-1 levels in all subjects compared to that during no medication. However, it is unclear why alogliptin alone or alogliptin and metformin co-administration resulted in lower GLP-1 levels in cases 1 and 2 compared with that when metformin alone was administered. Interestingly, GLP-1 levels $1 \mathrm{~h}$ after lunch, among all blood collection time points, were the highest in all subjects during alogliptin and metformin co-administration. One possible explanation for this observation is that metformin administration after lunch (the second dose of the day) may enhance the effect on metformin-induced GLP-1 secretion. Although GLP-1 levels after dinner were not examined in the present study, the results suggest that alogliptin and metformin co-administration, with metformin 3 times per day, may be beneficial for maintaining or even enhancing the elevation of postprandial GLP-1 levels, leading to attenuated postprandial blood glucose excursion. Total GIP levels in both cases 1 and 2 with alogliptin administration either alone or combined with metformin were slightly lower as compared with that during no medication. Meanwhile, it is unclear why GIP 
levels after breakfast were higher in the control subject with metformin alone as compared with that during no medication. As expected, the control subject exhibited no remarkable glucose fluctuations; therefore, caution should be exercised when assessing the levels of glycemic and incretin markers in control subject, because it remains uncertain how incretin hormones behave in normoglycemic condition.

Several studies have found that patients with type 2 diabetes exhibit attenuated GLP-1 secretion [23-26]. Our results were consistent with those findings, that is, subjects with diabetes had lower GLP-1 levels (even with alogliptin administration) than the control subject.

\section{Conclusion}

Using CGM, we showed that alogliptin and metformin coadministration is effective for reducing glucose fluctuations and stabilizing postprandial blood glucose levels. However, we cannot apply our results to the general population of patients with type 2 diabetes because only two patients were examined. The two patients had completely different patterns of insulin, glucagon, GLP-1, and GIP responses to the study medications, suggesting that hormonal responses to biguanides and DPP-4 inhibitors differ among individuals, and that these responses may be complicated by multiple factors. Further studies with larger numbers of patients are necessary to provide a more complete understanding of the hormonal responses induced by DPP-4 inhibitors and biguanides.

\section{Acknowledgement}

This work was supported by a grant from the Joint Research Association for Japanese Diabetes.

\section{Disclosure}

Miyako Kishimoto and Kaori Inoue declare that they have no conflicts of interest. Mitsuhiko Noda has received speaker honoraria from drug companies Dainippon Sumitomo Pharma, Daiichi Sankyo, MSD, Sanofi, and Novo Nordisk Pharma, and research grants from Daiichi Sankyo and Novartis Pharma.

\section{References}

1. Drucker DJ. The role of gut hormones in glucose homeostasis. J Clin Invest. 2007;117(1):24-32.

2. Meier JJ, Nauck MA. Incretins and the development of type 2 diabetes. Curr Diab Rep. 2006;6(3):194-201.

3. Mentlein R, Gallwitz B, Schmidt WE. Dipeptidyl- peptidase IV hydrolyses gastric inhibitory polypeptide, glucagon-like peptide-1(7-36)amide, peptide histidine methionine and is responsible for their degradation in human serum. Eur J Biochem. 1993;214(3):829-835.

4. Drucker DJ. Therapeutic potential of dipeptidyl peptidase IV inhibitors for the treatment of type 2 diabetes. Expert Opin Investig Drugs. 2003;12(1):87-100.

5. Stumvoll M, Nurjhan N, Perriello G, Dailey G, Gerich JE. Metabolic effects of metformin in non-insulin-dependent diabetes mellitus. N Engl J Med. 1995;333(9):550554.

6. Bailey CJ, Turner RC. Metformin. N Engl J Med. 1996;334(9):574-579.

7. Goldstein BJ, Feinglos MN, Lunceford JK, Johnson J, Williams-Herman DE. Effect of initial combination therapy with sitagliptin, a dipeptidyl peptidase-4 inhibitor, and metformin on glycemic control in patients with type 2 diabetes. Diabetes Care. 2007;30(8):1979-1987.

8. Deacon CF, Mannucci E, Ahren B. Glycaemic efficacy of glucagon-like peptide-1 receptor agonists and dipeptidyl peptidase-4 inhibitors as add-on therapy to metformin in subjects with type 2 diabetes-a review and meta analysis. Diabetes Obes Metab. 2012;14(8):762-767.

9. Scheen AJ. Pharmacokinetic and pharmacodynamic evaluation of sitagliptin plus metformin. Expert Opin Drug Metab Toxicol. 2010;6(10):1265-1276.

10. Kadowaki T, Tajima N, Odawara M, Nishi M, Taniguchi T, Ferreira JCA. Addition of sitagliptin to ongoing metformin monotherapy improves glycemic control in Japanese patients with type 2 diabetes over 52 weeks. J Diabetes Invest. 2013;4:174-181.

11. Gross TM, Bode BW, Einhorn D, Kayne DM, Reed JH, White NH, Mastrototaro JJ. Performance evaluation of the MiniMed continuous glucose monitoring system during patient home use. Diabetes Technol Ther. 2000;2(1):49-56.

12. Kaufman FR, Gibson LC, Halvorson M, Carpenter S, Fisher LK, Pitukcheewanont P. A pilot study of the continuous glucose monitoring system: clinical decisions and glycemic control after its use in pediatric type 1 diabetic subjects. Diabetes Care. 2001;24(12):2030-2034.

13. Sachedina N, Pickup JC. Performance assessment of the Medtronic-MiniMed Continuous Glucose Monitoring System and its use for measurement of glycaemic control in Type 1 diabetic subjects. Diabet Med. 2003;20(12):1012-1015.

14. Cho YM, Kieffer TJ. New aspects of an old drug: metformin as a glucagon-like peptide 1 (GLP-1) enhancer and sensitiser. Diabetologia. 2011;54(2):219-222.

15. Yasuda N, Inoue T, Nagakura T, Yamazaki K, Kira K, Saeki T, Tanaka I. Enhanced secretion of glucagon-like peptide 1 by biguanide compounds. Biochem Biophys Res Commun. 2002;298(5):779-784.

16. Mannucci E, Ognibene A, Cremasco F, Bardini G, Men- 
cucci A, Pierazzuoli E, Ciani S, et al. Effect of metformin on glucagon-like peptide 1 (GLP-1) and leptin levels in obese nondiabetic subjects. Diabetes Care. 2001;24(3):489-494.

17. Mannucci E, Tesi F, Bardini G, Ognibene A, Petracca MG, Ciani S, Pezzatini A, et al. Effects of metformin on glucagon-like peptide-1 levels in obese patients with and without Type 2 diabetes. Diabetes Nutr Metab. 2004;17(6):336-342.

18. Ahren B, Pacini G, Foley JE, Schweizer A. Improved meal-related beta-cell function and insulin sensitivity by the dipeptidyl peptidase-IV inhibitor vildagliptin in metformin-treated patients with type 2 diabetes over 1 year. Diabetes Care. 2005;28(8):1936-1940.

19. Cuthbertson J, Patterson S, O'Harte FP, Bell PM. Addition of metformin to exogenous glucagon-like peptide-1 results in increased serum glucagon-like peptide-1 concentrations and greater glucose lowering in type 2 diabetes mellitus. Metabolism. 2011;60(1):52-56.

20. Hinke SA, Kuhn-Wache K, Hoffmann T, Pederson RA, McIntosh $\mathrm{CH}$, Demuth HU. Metformin effects on dipeptidylpeptidase IV degradation of glucagonlike peptide-1. Biochem Biophys Res Commun. 2002;291(5):1302-1308.
21. Maida A, Lamont BJ, Cao X, Drucker DJ. Metformin regulates the incretin receptor axis via a pathway dependent on peroxisome proliferator-activated receptor-alpha in mice. Diabetologia. 2011;54(2):339-349.

22. Migoya EM, Bergeron R, Miller JL, Snyder RN, Tanen M, Hilliard D, Weiss B, et al. Dipeptidyl peptidase-4 inhibitors administered in combination with metformin result in an additive increase in the plasma concentration of active GLP-1. Clin Pharmacol Ther. 2010;88(6):801-808.

23. Vilsboll T, Krarup T, Deacon CF, Madsbad S, Holst JJ. Reduced postprandial concentrations of intact biologically active glucagon-like peptide 1 in type 2 diabetic patients. Diabetes. 2001;50(3):609-613.

24. Nauck M, Stockmann F, Ebert R, Creutzfeldt W. Reduced incretin effect in type 2 (non-insulin-dependent) diabetes. Diabetologia. 1986;29(1):46-52.

25. Toft-Nielsen MB, Madsbad S, Holst JJ. Determinants of the effectiveness of glucagon-like peptide-1 in type 2 diabetes. J Clin Endocrinol Metab. 2001;86(8):38533860 .

26. Rask E, Olsson T, Soderberg S, Johnson O, Seckl J, Holst JJ, Ahren B. Impaired incretin response after a mixed meal is associated with insulin resistance in nondiabetic men. Diabetes Care. 2001;24(9):1640-1645. 